\title{
Cúpulas de crucero en templos de la provincia de Alicant (s.XVII-XIX): construcción y geometría
}

\section{Crossing domes of temples in the province of Alicante (17th - 19th centuries): construction and geometry}

J. C. Pérez-Sánchez ${ }^{(*)}, \underline{B}$. Piedecausa-García ${ }^{(*)}$

\section{RESUMEN}

El objetivo es determinar las características constructivas, geométricas y materiales en la construcción de cúpulas originales de ladrillo en templos de la provincia de Alicante desde finales del siglo XVII hasta principios del siglo XIX. Se estudian 38 cruceros de iglesias y catedrales en 35 localidades alicantinas mediante recopilación de dibujos originales, levantamiento de planos, construcción en $3 \mathrm{D}$, termografías y toma de muestras, todo ello seguido de un análisis comparativo de variables. Constructivamente, el 88,57 \% de las cúpulas simples analizadas son de una hoja de ladrillo macizo (colocado a rosca). Geométricamente, el 97,37\% presentan planta circular y el 92,1\% peralte. El acabado azul vidriado en cubierta es el característico de la provincia $(68,42 \%)$, adornado con limatesas en color blanco.

Un estudio imprescindible para establecer criterios de uso y conservación, facilitando futuras intervenciones de rehabilitación en este tipo de construcciones religiosas.

Palabras clave: Cúpula de crucero; ladrillo; construcción religiosa; sistema constructivo; siglo XVII-XIX; patrimonio arquitectónico.

\section{ABSTRACT}

The main objective is to determine constructive, geometric and material properties in the construction of original brick domes of temples in the province of Alicante from the late seventeenth to the early nineteenth century. 38 crossing domes in 35 churches and cathedrals in Alicante municipalities were studied by collecting original drawings, draw up plans, $3 D$ construction, thermography and sampling, followed by a comparative analysis of variables. Constructively, the $88.57 \%$ of the analyzed simple domes are from a sheet of solid bricks. Geometrically, 97.37\% present circular floor and $92.1 \%$ have a camber shape. The blue glazed finish on decks is characteristic in the province (68.42\%), adorned with white easels.

This research is essential to establish use and conservation criteria, facilitating future rehabilitation interventions in such kind of religious buildings.

Keywords: Crossing dome; brick; religious construction; construction system; 17th-19th centuries; architectural heritage.

(*) Universidad de Alicante (España).

Persona de contacto/Corresponding author: piedecausa@ua.es (B. Piedecausa-García)

Cómo citar este artículo/Citation: Pérez-Sánchez, J. C., Piedecausa-García, B. (2015). Cúpulas de crucero en templos de la provincia de Alicante (s.XVII-XIX): construcción y geometría. Informes de la Construcción, 67(538): eo77, doi: http://dx.doi.org/10.3989/ic.13.176. Licencia/License: Salvo indicación contraria, todos los contenidos de la edición electrónica de Informes de la Construcción se distribuyen bajo una licencia de uso y distribución Creative Commons Reconocimiento no Comercial 3.o. España (cc-by-nc). 


\section{INTRODUCCIÓN}

Si hablamos de construcciones históricas, es indudable que las arquitecturas religiosas son muy representativas por su carácter monumental, por albergar la evolución de formas y métodos constructivos a lo largo de siglos, por su importancia en la historia de la humanidad y por el empeño volcado en ellas por distintas civilizaciones. Así, dentro de esta arquitectura, es en los templos donde emergen infinidad de elementos constructivos, innovadores en su época, cuyo conocimiento requiere un análisis en profundidad.

Los primeros estudios españoles sobre la construcción y geometría de construcciones abovedadas, donde se incluyen las cúpulas, se encuentran en tratados de los siglos XVII, XVIII y XIX (1) (2). Muchos de ellos describen aspectos más geométricos que constructivos, y proponen reglas empíricas para el dimensionado de distintos elementos, muchas veces repetidas posteriormente.

En la segunda mitad del siglo XIX, aparecen publicaciones sobre historia de la construcción en estructuras abovedadas (3) (4) (5) (6); y, a partir del siglo XX, aunque esta construcción estaba en desuso, siguieron apareciendo estudios relacionados con sus técnicas constructivas (7) (8).

Por una parte, son pues muchos los análisis generalistas desarrollados históricamente sobre los sistemas abovedados, tanto de temática general como local. No obstante, aquellos que guardan relación directa con el ámbito constructivo son escasos, obedeciendo más a aspectos históricos, artísticos, gráficos o de cálculo. Por otra, al localizar esta propuesta en la provincia de Alicante (España), existen ciertos estudios relacionados con el ámbito de las cúpulas; sin embargo, ninguno de ellos analiza de lleno su planteamiento constructivo y se centran bien en su intervención/conservación (9), o bien en su geometría y modelo de trazado (10).

\section{OBJETIVOS}

El objetivo principal de la investigación es determinar las características constructivas, geométricas y materiales en la construcción de cúpulas de ladrillo en los templos de la provincia de Alicante desde finales del siglo XVII hasta principios del XIX, con el fin de proporcionar un conocimiento riguroso y de utilidad en estudios posteriores e intervenciones para su conservación.

Dentro de la gran variedad de elementos constructivos de los templos, el estudio se delimita en su cobertura y se particulariza en la cúpula, su protagonista arquitectónico indiscutible. $\mathrm{Al}$ exterior, este elemento resalta sobre el paisaje, integrándose y convirtiéndose en símbolo común a todos los pueblos; al interior, despliega su grandeza, resultado de una gran hazaña con medios, materiales y presupuesto muy ajustados, de los que, hoy en día, se tiene un legado muy escaso.

\section{1. Ámbito temporal y espacial}

El ámbito temporal y espacial de la investigación se enmarca en la costa mediterránea, concretamente en la provincia de Alicante, desde finales del siglo XVII a principios del siglo XIX; siendo el siglo XVIII cuando se produce el máximo esplendor en la construcción de templos, con la cúpula como elemento característico.

Dichos márgenes temporales no están referenciados a consideraciones historiográficas sino constructivas, ya que a fina- les del XVII se pasa de la piedra al uso mayoritario del ladrillo en las cúpulas de la provincia. A partir del segundo tercio del XIX empiezan a utilizarse nuevos materiales: paulatinamente el ladrillo macizo se alterna con ladrillo hueco o rasilla, el yeso será sustituido total o parcialmente como conglomerante por mortero de cemento y el acero será utilizado habitualmente para dar estabilidad a la cúpula, tanto en nueva construcción como en restauraciones.

También, destaca como criterio de selección espacial aquel identificado geográficamente con la provincia de Alicante, aunque no coincida con el límite de la diócesis Orihuela-Alicante, perteneciendo algunos templos a la Archidiócesis de Valencia.

\subsection{Selección de templos}

Para la selección de cúpulas, se ha partido del inventario realizado por García Jara (10) sobre 159 templos en la provincia de Alicante, donde se incluyen iglesias, ermitas, monasterios y otros edificios religiosos, bien de piedra o ladrillo, y se analizan falsas cúpulas o totalmente restauradas.

En base a este inventario, se realizó una selección propia atendiendo a distintos criterios como el empleo del ladrillo, el ámbito temporal (desde finales del siglo XVII a principios del XIX) y el ámbito espacial (la provincia de Alicante). Se eligen cúpulas correspondientes a iglesias o catedrales en elementos de cruceros, no incluyendo ermitas ni otras construcciones religiosas. No se incluyen cúpulas de menor entidad como las localizadas en capillas, camarines o naves laterales, ni las falsas cúpulas (normalmente con cañizo y yeso) ya que no se comportan constructivamente como tal. Por último, cabe destacar que las cúpulas elegidas son históricas, es decir originales, y no han sido restauradas en su totalidad; son válidas aquellas que, aunque intervenidas, han mantenido su estructura de ladrillo original.

Teniendo en cuenta estas consideraciones, el presente estudio realiza una selección final de 38 cúpulas de cruceros, pertenecientes a templos ubicados en 35 localidades de la provincia de Alicante (Tabla 1 y Figura 1).

\section{METODOLOGÍA}

La metodología planteada se centra en una primera investigación sobre documentación específica de cada templo analizado (mediante toma de datos in situ o revisión de fuentes documentales); una segunda parte de relación, análisis y comparativa de los datos individualmente obtenidos con otros estudios similares; y, finalmente, el establecimiento de conclusiones definitorias y generalizables a la construcción de cúpulas de ladrillo en los templos de la provincia de Alicante desde finales del siglo XVII hasta principios del XIX. Los métodos utilizados en el transcurso de la investigación son descritos a continuación.

\subsection{Toma de datos}

\subsubsection{Datos in situ}

Durante la toma de datos en los templos se ha seguido un método ordenado y riguroso que ofreciera garantías en la obtención de resultados. Para el acceso a documentación 
Tabla 1. Templos donde se analiza la cúpula de crucero. (Fuente propia).

\begin{tabular}{|c|c|c|c|c|c|c|c|}
\hline No & LOCALIDAD & TEMPLO & FECHA & No & LOCALIDAD & TEMPLO & FECHA \\
\hline $\mathbf{1}$ & AGOST & Iglesia de San Pedro Apóstol & s. XVIII & 20 & DENIA & $\begin{array}{l}\text { Iglesia Arciprestal de la } \\
\text { Asunción }\end{array}$ & ca. 1755 \\
\hline 2 & $\begin{array}{l}\text { AGUAS DE } \\
\text { BUSOT }\end{array}$ & $\begin{array}{l}\text { Iglesia de San Francisco de } \\
\text { Asís }\end{array}$ & $1745-1755$ & 21 & DOLORES & $\begin{array}{l}\text { Iglesia de la Virgen de los } \\
\text { Dolores }\end{array}$ & ca. 1770 \\
\hline 3 & ALBATERA & Iglesia de Santiago Apóstol & 1729 & 22 & FINESTRAT & Iglesia de San Bartolomé & s. XVIII \\
\hline 4 & \multirow{2}{*}{ ALICANTE } & Monasterio de la Santa Faz & ca. 1765 & 23 & $\begin{array}{l}\text { GRANJA DE } \\
\text { ROCAMORA }\end{array}$ & Iglesia de San Pedro Apóstol & s. XVIII \\
\hline 5 & & $\begin{array}{l}\text { Iglesia de San José } \\
\text { (Villafranqueza) }\end{array}$ & ca. 1785 & 24 & $\begin{array}{l}\text { HONDÓN DE } \\
\text { LAS NIEVES }\end{array}$ & $\begin{array}{l}\text { Iglesia de la Virgen de las } \\
\text { Nieves }\end{array}$ & ca. 1757 \\
\hline 6 & ASPE & Iglesia de la Virgen del Socorro & ca. 1735 & 25 & IBI & $\begin{array}{l}\text { Iglesia de la Transfiguración } \\
\text { del Señor }\end{array}$ & s. XVIII \\
\hline 7 & $\begin{array}{l}\text { BANYERES DE } \\
\text { MARIOLA }\end{array}$ & Iglesia de Santa María & s. XVIII & 26 & JALÓN & Iglesia de Santa María & ca. 1828 \\
\hline 8 & BENEIXAMA & Iglesia de San Juan Bautista & ca. 1830 & 27 & $\begin{array}{l}\text { MONFORTE } \\
\text { DEL CID }\end{array}$ & $\begin{array}{l}\text { Iglesia de la Virgen de las } \\
\text { Nieves }\end{array}$ & s. XVIII \\
\hline 9 & BENIDORM & $\begin{array}{l}\text { Iglesia de San Jaime y Santa } \\
\text { Ana }\end{array}$ & ca. 1780 & 28 & MONÓVAR & Iglesia de San Juan Bautista & ca. 1756 \\
\hline 10 & BIAR & $\begin{array}{l}\text { Santuario de la Virgen de } \\
\text { Gracia }\end{array}$ & s. XVIII & 29 & MUTXAMEL & Iglesia del Salvador & s. XVIII \\
\hline 11 & $\begin{array}{l}\text { CALLOSA } \\
\text { D’EN SARRIÁ }\end{array}$ & Iglesia de San Juan Bautista & ca. 1780 & 30 & NOVELDA & Iglesia de San Pedro & s. XVIII \\
\hline 12 & \multirow{2}{*}{$\begin{array}{l}\text { CALLOSA DE } \\
\text { SEGURA }\end{array}$} & $\begin{array}{l}\text { Iglesia Arciprestal de San } \\
\text { Martín }\end{array}$ & ca. 1683 & 31 & \multirow{2}{*}{ ORIHUELA } & Iglesia del Carmen & $1730-1740$ \\
\hline $\mathbf{1 3}$ & & $\begin{array}{l}\text { Iglesia de la Virgen de los } \\
\text { Dolores }\end{array}$ & s. XVIII & 32 & & $\begin{array}{l}\text { Iglesia de Nuestra Señora de } \\
\text { Monserrate }\end{array}$ & s. XVIII \\
\hline 14 & $\begin{array}{l}\text { CAMPO DE } \\
\text { MIRRA }\end{array}$ & Iglesia de San Bartolomé & s. XVIII & 33 & PETRER & Iglesia de San Bartolomé & s. XVIII-XIX \\
\hline 15 & CAÑADA & Iglesia de San Cristóbal & s. XVIII & 34 & PINOSO & Iglesia de San Pedro Apóstol & s. XVIII \\
\hline 16 & CATRAL & Iglesia de los Santos Juanes & ca. 1786 & 35 & SALINAS & Iglesia de San Antonio Abad & s. XVIII \\
\hline 17 & COCENTAINA & Iglesia de Santa María & ca. 1668 & 36 & \begin{tabular}{|l|} 
SAN \\
FULGENCIO
\end{tabular} & Iglesia de San Fulgencio & ca. 1760 \\
\hline 18 & $\operatorname{cox}$ & Iglesia de San Juan Bautista & ca. 1770 & 37 & \begin{tabular}{|l|} 
SAN VICENTE \\
DEL RASPEIG \\
\end{tabular} & Iglesia de San Vicente Ferrer & s. XVIII \\
\hline 19 & CREVILLENTE & Iglesia de la Virgen de Belén & ca. 1828 & 38 & TIBI & $\begin{array}{l}\text { Iglesia de Santa María } \\
\text { Magdalena }\end{array}$ & s. XVIII \\
\hline
\end{tabular}

in situ fue necesario establecer permisos de cada responsable, concretando convenientemente fecha y hora de las visitas necesarias. Así, se ha inspeccionado detenidamente cada edificio, realizando infinidad de croquis a mano alzada acotados (plantas, secciones y detalles) mediante distanciómetro laser Leica DISTO, flexómetro y cinta métrica (Figura 2). Esta tarea ha sido imprescindible y supone la generación de una documentación propia con incalculable valor ya que, aunque algunos templos cuenten con información gráfica, en muchos casos el material no se ajusta a la realidad o no tiene el rigor suficiente para su estudio con detalle, principalmente las secciones, al no reflejar la realidad constructiva.

Paralelamente a esta labor, para cada templo se ha elaborado una base de datos con información relativa a los elementos del cuerpo central cubierto por cúpulas. Las variables tenidas en cuenta en el análisis de datos son: dimensiones generales (altura total cúpula, altura total linterna, diámetro cúpula, espesor muros y área cúpula), columnas (área/ud., altura total y altura basa), estribos (longitud, espesor y altura), arcos torales (flecha, diámetro 1, diámetro 2 y altura total), pechinas (arranque,
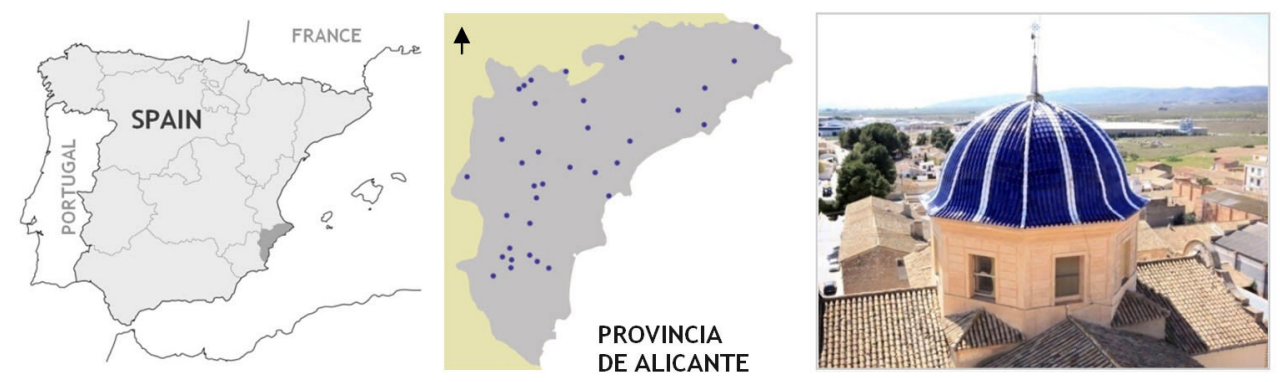

Figura 1. Ubicación de los 38 templos analizados, situados en 35 municipios de la provincia de Alicante y ejemplo de cúpula de crucero de la Iglesia de San Juan Bautista, en Beneixama.

(Fuente propia). 

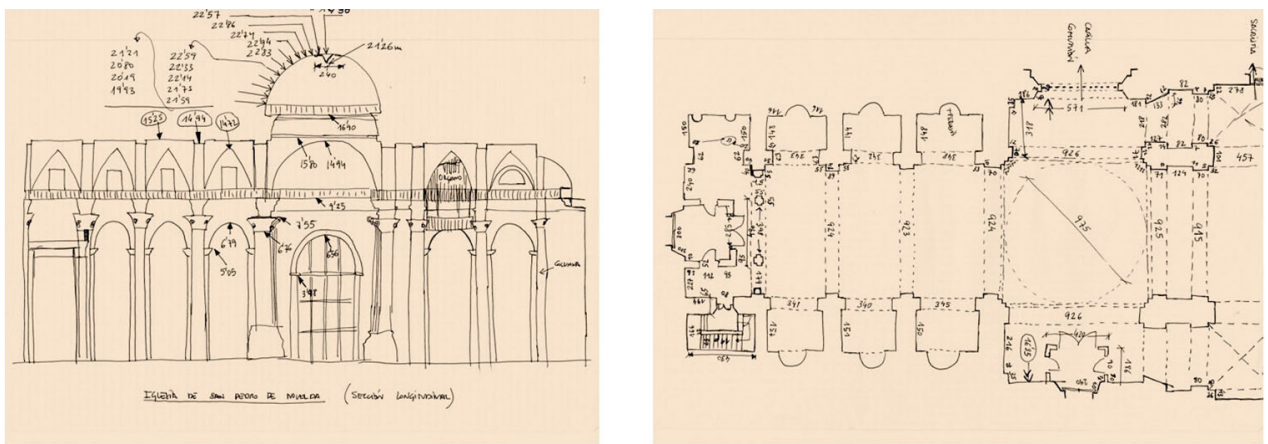

Figura 2. Sección y planta de Iglesia de San Pedro en Novelda. (Fuente propia).

final, diámetro, longitud arranque y retranqueo), tambor (altura interior, diámetro interior, espesor, forma planta interior y exterior, número de nervios interiores y número de huecos), cúpula (número de hojas, flecha interior, diámetro interior, flecha/diámetro, número de nervios interiores, tipo y color de limatesas exteriores, forma y número de lados en aleros, forma y número de huecos), linterna (dimensiones, número de nervios interiores y exteriores, número y forma de huecos).

Estas variables geométricas han sido completadas con documentación complementaria para cada ejemplo analizado, a saber:

- Fotografías digitales interiores, exteriores y de detalle (más de 15.000) para la identificación de elementos constructivos inaccesibles o inapreciables mediante cámara réflex digital CANON EOS-450D, objetivos electrónicos SIGMA 10-20 mm, CANON 18-55 mm, CANON 55-200 mm, filtros UV y trípode VELBON.

- Termografías interiores y exteriores de la totalidad de las cúpulas (más de 500) como fuente de información adicional en ciertos casos que ha permitido determinar características de interés como el sistema constructivo (si la cúpula es tabicada o a rosca), la colocación de distintos elementos o la identificación de sistemas de refuerzo estructural (si han sido restauradas con estructuras metálicas auxiliares), mediante cámara termográfica TermaCAM P25 de FLIR (Figura 4).

- Toma de muestras de materiales intervinientes en el proceso constructivo, como son ladrillos y tejas. Se ha analizado un total de 16 muestras de ladrillos macizos (correspondientes a templos diferentes) y 10 muestras de tejas curvas (ídem).

- Planos de plantas, secciones y detalles en 3D. Tras los croquis a mano alzada, y con la ayuda de las fotografías, se ha procedido al levantamiento de planos para cada templo mediante el programa AutoCAD (Figura 3). Esta documentación no solo ha servido para el estudio geométrico de sus elementos, sino para dar respuesta a muchas cuestiones relativas a procesos, formas y justificación de soluciones constructivas.

\subsubsection{Fuentes documentales}

Paralelamente a la obtención de datos in situ, se ha recopilado toda información documental existente, tanto de carácter general como específico, para cada templo estudiado.

Respecto a la documentación general, se ha realizado un profundo vaciado bibliográfico, siendo muchas las fuentes históricas escritas aunque escasas las que tratan con detalle la construcción de elementos abovedados (1) (2) (11) (12) (13). Por otro lado, respecto a la documentación específica, se han visitado archivos parroquiales (muchos destruidos durante la guerra civil), archivos municipales (con proyectos de restauración y fotografías del proceso) (Figura 4) y otros archivos de asociaciones, cronistas, fotógrafos, arquitectos, arquitectos técnicos o empresas constructoras que se han interesado o intervenido en la conservación y restauración de los templos (mediante entrevistas o el estudio de documentación gráfica).

En cuanto al material conservado en archivos, destaca la memoria de Miguel Francia (15), posteriormente arquitecto de la diócesis de Orihuela, para la Iglesia de los Santos Juanes de Catral, que ha sido de gran utilidad para el estudio de las cúpulas, al incluir distintos capítulos con una memoria explicativa de cómo realizar la nueva obra de la iglesia.
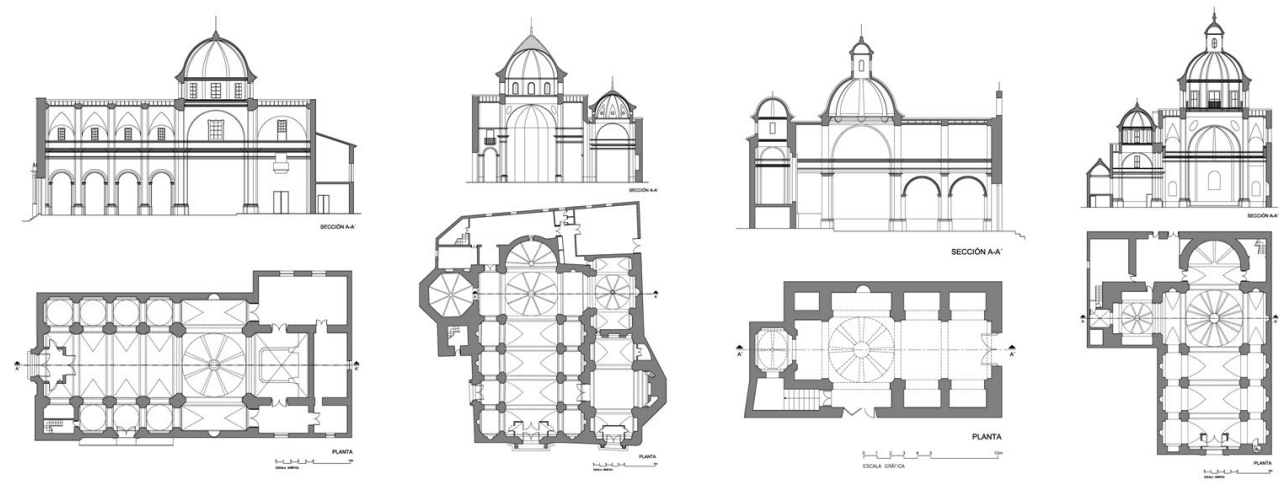

Figura 3. Secciones y plantas de cuatro tempos representativos de la provincia de Alicante. De izquierda a derecha, Iglesia de la Virgen de los Dolores en Dolores, Iglesia de los Santos Juanes en Catral, Iglesia de la Virgen de los Dolores en Callosa del Segura e Iglesia de San Juan Bautista en Cox. (Fuente propia). 

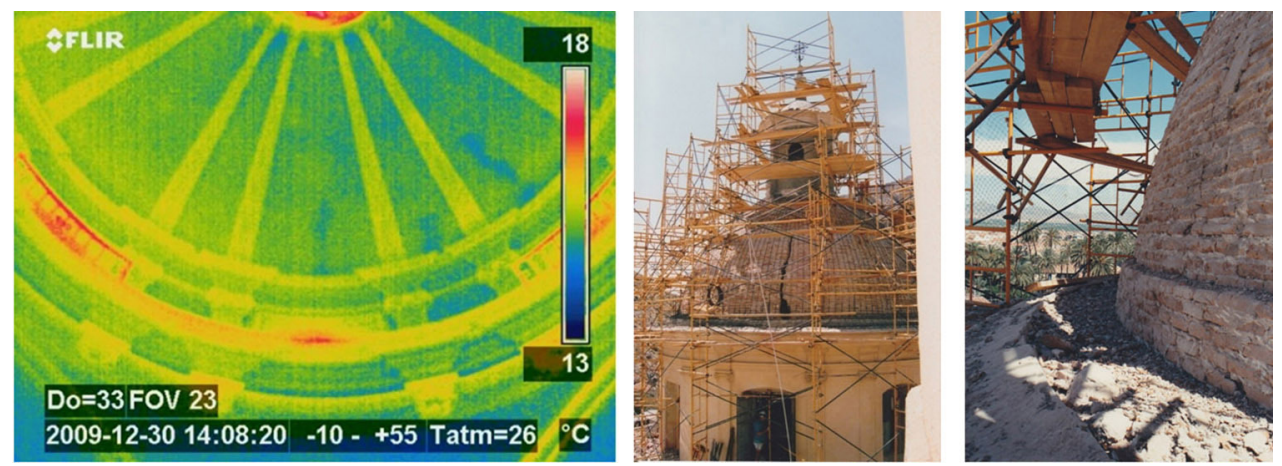

Figura 4. Documentación obtenida de la Iglesia de San Juan Bautista en Cox. De izquierda a derecha, termografía interior de la cúpula y vistas exteriores durante la restauración. (Fuente: la primera imagen es propia; la segunda y tercera imagen son fotos del Archivo Parroquial de San Juan Bautista de Cox (13).

\subsection{Método de análisis}

La relación de los datos anteriores ha permitido el completo conocimiento de las cúpulas tanto en su visión general como particular, siendo el estudio de fotografías y termografías determinante para comprender el proceso constructivo de cada cúpula; la puesta a escala de los croquis en cada templo, junto con la realización de dibujos en $3 \mathrm{D}$, ha permitido completar el estudio.

Con todos estos datos, y para obtener resultados relativos a sus materiales, geometría y construcción, se ha tratado la información considerando una muestra con 38 cúpulas de crucero en 35 municipios diferentes de la provincia de Alicante.

Por una parte, en cuanto a la geometría, se han obtenido valores dimensionales medios de la cúpula y los elementos constructivos que guardan relación con ésta. Además, se han establecido relaciones entre pares de variables, buscando reglas de dimensionado que han sido comparadas con otros estudios.

Por otra, respecto a la construcción, se han reproducido de manera pormenorizada todas las cúpulas, con las distintas soluciones encontradas. Para ello, se han realizado multitud de dibujos en 3D representando, ladrillo a ladrillo, las distintas formas de construcción y elementos de sustento encontrados; también se han comparado los resultados y se han establecido relaciones referentes al diseño, materiales y evolución de los sistemas constructivos.

\section{RESULTADOS}

\subsection{Sistemas constructivos de las cúpulas de crucero}

En la construcción de templos, la cúpula es el último elemento en ejecución, siendo necesario levantar previamente muros, contrafuertes y machones que transmiten las cargas al terreno a través de la cimentación, así como arcos fajones y bóvedas que cubren los espacios del crucero. Por ello, el presente estudio de cúpulas conlleva la necesidad de un análisis previo de todos los elementos constructivos que participan directa o indirectamente en su construcción y equilibrio.

De este modo, en la Figura 5 se muestran, de forma resumida y ordenada según su ejecución, las fases del proceso constructivo de la cúpula del crucero donde, una vez erigidos los muros, machones y estribos, se construyen los arcos torales y bóvedas, las pechinas y anillo que enlazan con ellos, el tambor y la cornisa, la cúpula y su contrarresto inferior $\mathrm{y}$, finalmente, la cubierta; esta última, con el característico perfil contracurvo típico en los templos de la provincia.

\subsubsection{Arcos torales}

Los arcos torales situados en el crucero, desde los que parten las bóvedas de cañón, son sobre los que se sustenta la cúpu-
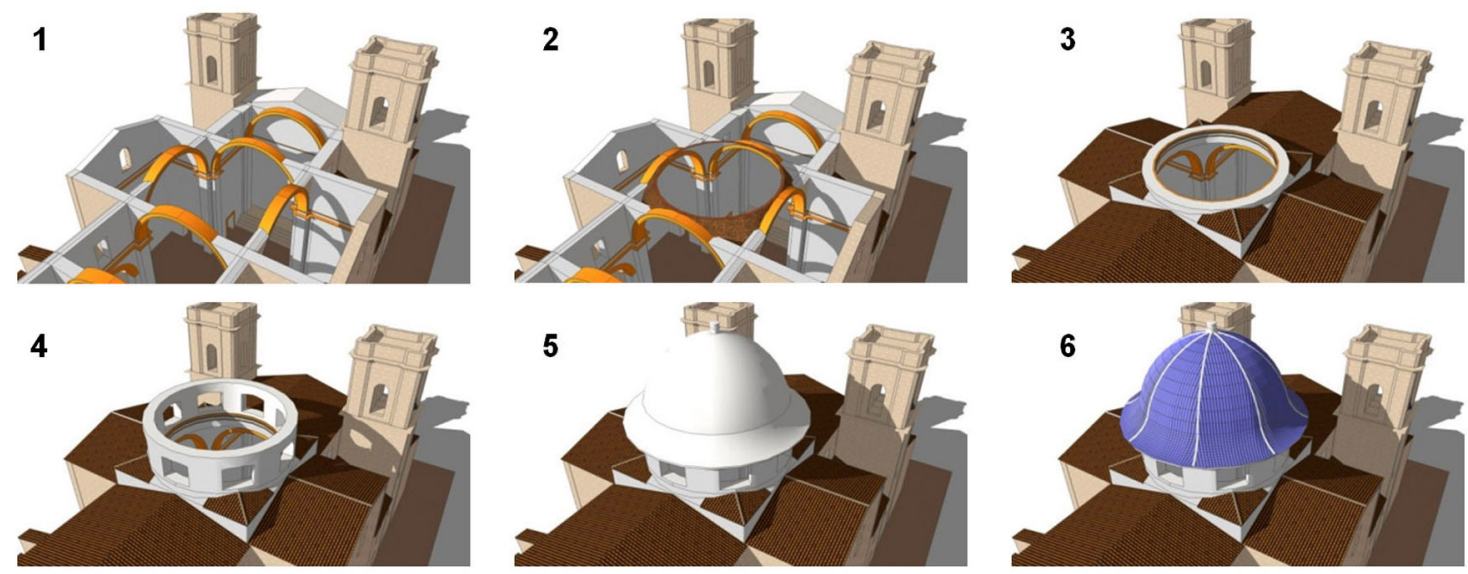

Figura 5. Fases del proceso constructivo de la cúpula de crucero: 1 Arcos torales. 2 Pechinas. 3 Anillo. 4 Tambor. 5 Cúpula. 6 Cubierta. (Fuente propia). 
la. En la mayoría de los templos están construidos con ladrillo (siendo escasos aquellos con dovelas de piedra, más habituales en épocas anteriores) y son de perfil semicircular, es decir, presentan una relación de $1 / 2$ entre la flecha y el diámetro, con un espesor de 2 sogas de ladrillo y un diámetro medio de 7,86 m. La organización constructiva más habitual es de aparejo a rosca de ladrillo y yeso. En ocasiones, se emplean de uno a tres gruesos de ladrillo y yeso tabicados a modo de encofrado perdido sobre el que se construye el arco resistente a rosca.

Además, se ha constatado que, independientemente del material con que fueran construidos, en todos los ejemplos estudiados fue necesario el empleo de cimbras simétricas durante su ejecución para repartir las cargas correctamente y evitar deformaciones; así como la existencia, en todos los casos, de enjarjes laterales en contacto con las pechinas para entrelazar los movimientos de ambos.

\subsubsection{Pechinas}

Para su construcción en los templos alicantinos se emplean fábricas de ladrillo y yeso; esta fábrica queda oculta bajo el revestimiento interior de yeso y, sobre él, normalmente se colocan lienzos de los cuatro evangelistas. La transición del cuadrado definido por los arcos torales al círculo del anillo se realiza mediante el empleo de pechinas, que presentan dos formas de acometer el arranque (de boquilla o de rincón), condicionando la forma constructiva de proceder. Por una parte, cuando las pechinas arrancan de boquilla (60,53\%), se construyen por hiladas horizontales de ladrillos colocados a soga volados sobre la hilada inferior, en una planta de corona circular, constituyendo una falsa cúpula en saledizo, de forma que la unión con el anillo no plantea ningún problema. Por otra, cuando arrancan de rincón (39,47 \%), se construyen por hiladas de ladrillo colocados a sardinel, teniendo que ajustar la última hilada a la geometría del anillo. En ambas situaciones, las pechinas quedan unidas con los arcos torales y con el anillo superior mediante enjarjes. En todos los casos se constata que la construcción de pechinas se retranquea de media $13 \mathrm{~cm}$ respecto al intradós del arco toral, garantizando la correcta transmisión de empujes entre ambos elementos.

\subsubsection{Anillo}

La misión del anillo horizontal es unir arcos torales y pechinas para que trabajen conjuntamente, empleando fábricas de mampostería o ladrillo (siendo mayoritario este último en las restauraciones estudiadas). Así, los templos de la provincia podrán tener tambor o no, pero siempre tienen anillo circular por su parte interior, tangente a los arcos torales, que salva el desnivel entre los faldones de cubierta. La organización constructiva y el espesor del anillo coincide con los del tambor, aunque en algunos casos aumenta unos $15 \mathrm{~cm}$ (es decir, la dimensión del tizón del ladrillo) facilitando el reparto de cargas.

Por su parte exterior, el anillo puede ser circular u octogonal, siendo mayoritario este último en un $71,15 \%$. Su ancho puede coincidir con el del arco toral, en cuyo caso es tangente por su parte exterior, o puede ser algo menor, en cuyo caso quedará enrasado al interior.

\subsubsection{Tambor}

Una vez ejecutado el anillo se construye el tambor mediante fábricas de piedra (mampostería), ladrillo (hiladas hori- zontales de ladrillo colocado a soga o a tizón según el caso) o mixtas, utilizando como conglomerante mortero de cal; aunque puede no existir tambor, apareciendo directamente la cúpula.

De los casos analizados, 28 cúpulas presentan tambor, con un espesor medio en sus muros de $0,82 \mathrm{~m}$, un valor correspondiente a 1/10,95 del diámetro de la cúpula; esta relación es muy similar a la fijada por Fontana (16) en 1/10, aunque bastante inferior a los valores de García Jara (10) quien presenta espesores medios de 1/7,14 del diámetro de la cúpula, y alturas medias de $2 / 3$ de dicho diámetro.

De los 24 casos analizados donde existen huecos en el tambor, el 91,66 \% presenta 8 huecos que aligeran la estructura y proporcionan iluminación interior, dando más esbeltez al elemento. En cuanto a sus dimensiones, en el $100 \%$ de casos el diámetro del tambor coincide con el de la cúpula (estando ésta enrasada por su parte interior) y su altura es considerable (una altura interior media de 3,39 m), alcanzando una proporción de 1/8,77 respecto a la altura de la cúpula. En función del diámetro, la altura del tambor es 1/3,34 el diámetro de la cúpula; una relación un poco inferior a la fijada por Fontana (15) en 1/2.

\subsubsection{Cúpula}

Según Huerta (17), a lo largo de la historia se han construido cúpulas tabicadas muy delgadas en España, con espesores mínimos de $1 / 100$ del radio de curvatura, presentando siempre rellenos y lengüetas en su trasdós hasta $2 / 3$ de su altura. Sin embargo, los templos seleccionados muestran espesores medios de $0,15 \mathrm{~m}$ en la zona superior, suponiendo un espesor máximo de $1 / 40$ del radio de curvatura. El empuje radial de todas las cúpulas está contrarrestado en la provincia por un sistema de estribos en su zona inferior mediante el relleno de enjutas con mortero de cal y cascotes, generando una forma contracurva al exterior, macizada siempre simétricamente.

Siguiendo la clasificación de Fornés y Gurrea (2) para las cúpulas de fábrica de ladrillo, se plantea la catalogación constructiva de las cúpulas alicantinas estudiadas en dos grupos:

\section{Según las hojas que las componen y su colocación (una o dos hojas)}

En los templos de la provincia se han empleado cáscaras de una sola hoja (colocada a rosca) y de dos hojas (tabicadas) (Figura 6). De las 35 cúpulas simples incluidas en el estudio, el 88,57 \% están compuestas por una sola hoja a rosca. Respecto a las 3 cúpulas dobles analizadas, y teniendo en cuenta que los datos disponibles son de la cáscara interior, existe un ejemplo de cáscara a rosca y dos tabicadas.

En los casos analizados, las cúpulas a rosca se construyen con un ladrillo colocado a soga y con argamasa de yeso, permitiendo su ejecución sin cimbras o con cimbras muy ligeras, mediante hiladas con juntas perfectamente trabadas. Las cúpulas tabicadas están construidas por dos gruesos de ladrillo colocados a panderete con unos $10 \mathrm{~cm}$ de espesor total, incluyendo la capa intermedia de conglomerante pasta de yeso. En este caso, la esbeltez máxima (relación entre espesor y radio de curvatura) es de $1 / 60$, es decir, un espesor muy pequeño, algo menor al de las cúpulas construidas con ladrillo a rosca. 


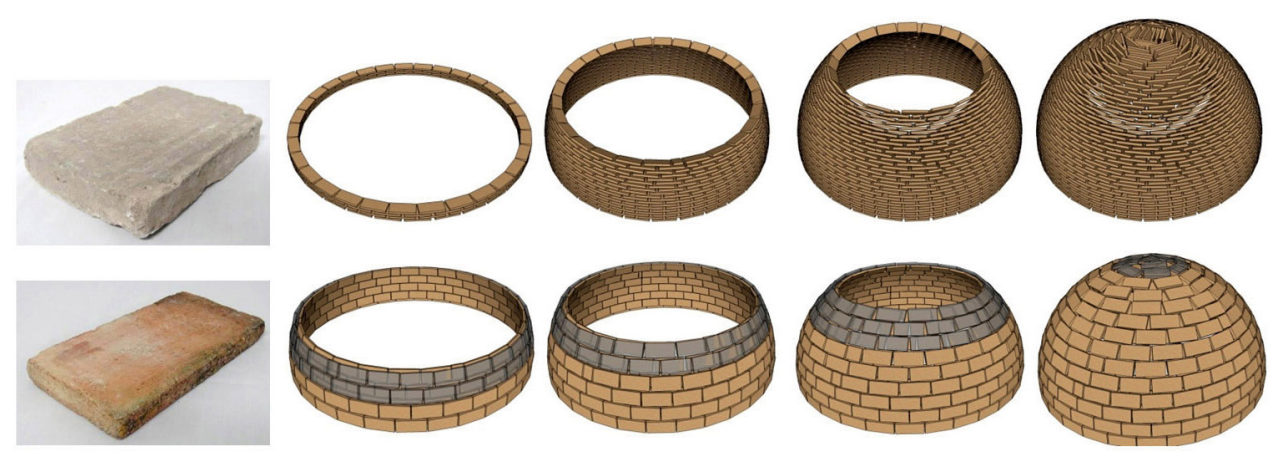

Figura 6. Línea superior. Muestra de ladrillo macizo tamaño grande de $30 \times 15 \times 3,5 \mathrm{~cm}$ y $2,5 \mathrm{~kg}$. Proceso constructivo de Cúpula construida con ladrillo a rosca. (Fuente propia).

Línea inferior. Muestra de ladrillo macizo tamaño pequeño de $26 \times 13 \times \mathrm{cm}$ y 1,5 kg. Proceso constructivo de Cúpula de ladrillo tabicada. (Fuente propia).

\section{Según el número de cáscaras (simples o dobles)}

En los templos analizados se encuentran cúpulas tanto con una cáscara (simples) como con dos cáscaras separadas entre sí (dobles). Las cúpulas simples son las más abundantes dada su sencillez de ejecución y mayor economía $(92,11 \%)$, construyéndose bien con ladrillos a rosca $(15 \mathrm{~cm}$ de espesor de cúpula) o bien tabicadas con dos hojas de ladrillo (10 cm de espesor de cúpula). Las cúpulas dobles se ejecutan desde fuera hacia adentro, con el perfil exterior más peraltado que se aleja de la geometría de la cáscara interior.

\subsubsection{Cubierta}

En función del alero existente, definido por la forma en planta del tambor, el arranque de la cúpula puede ser octogonal (71,05 \%) (Figura 7), en cuyo caso las limatesas coinciden con los vértices del octógono, circular (26,32 \%) o elíptico $(2,63 \%)$; los tres procedimientos de construcción son similares, diferenciándose únicamente en la transición del alero, siendo las circulares las más sencillas de ejecutar.

Una vez construida la cúpula, el remate exterior se ejecuta mediante cobertura de teja curva, dividiendo la superficie en faldones o gajos. En los templos de estudio, habitualmente existen 8 limatesas $(86,84 \%)$, siendo pocos los casos donde se duplican a $16(10,53 \%)$ y un único caso con 6 elementos (2,63\%). Si el tambor es octogonal, las limatesas coinciden con los vértices del octógono, y si es circular, se colocan coincidiendo con las pilastras exteriores entre los huecos.

\subsection{Geometría de las cúpulas de crucero}

En este apartado se estudian las cúpulas de crucero desde el punto de vista de su geometría, comparando las dimensiones obtenidas.

\subsubsection{Forma en planta}

En los templos seleccionados se desarrollan dos tipos de cúpulas según su forma en planta: circulares y elípticas. El trazado circular no presenta dificultad y viene definido por el anillo o tambor, cuyo centro coincide con el de la circunferencia de arranque de la cúpula. El trazado elíptico tampoco presenta dificultad en planta, aunque genera más problemas de ejecución al necesitar cimbras, no tanto para sustentar la cúpula sino para guiar su construcción y conseguir el peralte deseado, siendo una solución poco adoptada.

Prácticamente la totalidad de los ejemplos estudiados presentan cúpulas de crucero de planta circular, tanto si arrancan sobre anillo como sobre tambor, encontrando un único caso con forma elíptica; sin embargo, y aunque la construcción elíptica sea minoritaria en cruceros por su mayor dificultad de ejecución, existen bastantes ejemplos en la provincia en cúpulas de capillas con menores dimensiones (futuro objeto de publicación de los mismos autores).

\subsubsection{Forma del perfil interior y exterior}

Las 38 cúpulas analizadas son mayoritariamente peraltadas (92,10 \%), curiosamente las más complejas de construir y las de mayor interés para el estudio, siendo muy escasos los ejemplos con perfil rebajado $(7,80 \%)$ o semiesférico $(0,10 \%)$.

$\mathrm{Al}$ interior, la cúpula queda revestida con yeso y adornada con una serie de nervios decorativos. De los templos analizados, un 23,68 \% no poseen ningún nervio, un 2,63\% muestra 4 nervios, un $44,74 \%$ presenta 8 nervios y un $28,95 \%$ tiene 16 nervios (8 nervios dobles).

\subsubsection{Dimensiones y relaciones geométricas}

En cuanto a las dimensiones generales de las cúpulas, referenciadas interiormente, destacan como valores medios: altura
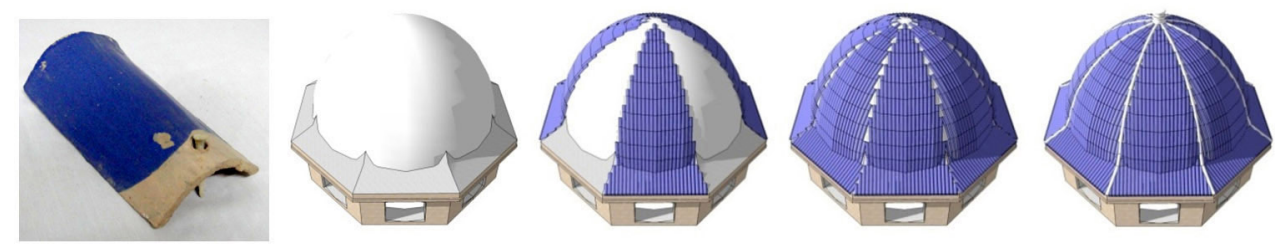

Figura 7. Muestra de teja curva. Proceso constructivo de cubierta en cúpula con alero octogonal. (Fuente propia). 
Tabla 2. Valores medios obtenidos de altura, diámetro y flecha de cúpula en los templos seleccionados. Fuente: propia. Leyenda: $\mathrm{A}(\mathrm{m})=$ altura de cúpula en metros. $\mathrm{D}(\mathrm{m})=$ diámetro de cúpula en metros. $\mathrm{F}(\mathrm{m})=$ flecha de cúpula en metros.

\begin{tabular}{|c|c|c|c|c|c|c|c|c|c|c|c|c|c|c|c|}
\hline & $\mathbf{a}(\mathbf{m})$ & $\mathbf{d ~ ( m )}$ & $\mathbf{f}(\mathbf{m})$ & & $\mathbf{a}(\mathbf{m})$ & $\mathbf{d ~ ( m )}$ & $\mathbf{f}(\mathbf{m})$ & & $\mathbf{a}(\mathbf{m})$ & $\mathbf{d ~ ( m )}$ & $\mathbf{f}(\mathbf{m})$ & & $\mathbf{a}(\mathbf{m})$ & $\mathbf{d}(\mathbf{m})$ & $\mathbf{f}(\mathbf{m})$ \\
\hline $\mathbf{0 1}$ & 27,10 & 9,30 & 5,66 & $\mathbf{1 1}$ & 28,04 & 11,21 & 4,74 & $\mathbf{2 1}$ & 23,52 & 8,52 & 5,02 & $\mathbf{3 1}$ & 15,83 & 6,02 & 3,46 \\
\hline $\mathbf{0 2}$ & 17,83 & 6,12 & 3,89 & $\mathbf{1 2}$ & 24,65 & 8,85 & 4,80 & $\mathbf{2 2}$ & 19,76 & 7,32 & 5,29 & $\mathbf{3 2}$ & 23,63 & 9,65 & 6,13 \\
\hline $\mathbf{0 3}$ & 27,84 & 10,02 & 6,34 & $\mathbf{1 3}$ & 11,47 & 4,89 & 2,91 & $\mathbf{2 3}$ & 24,36 & 7,79 & 5,05 & $\mathbf{3 3}$ & 24,89 & 9,53 & 4,46 \\
\hline $\mathbf{0 4}$ & 27,65 & 8,50 & 6,01 & $\mathbf{1 4}$ & 20,50 & 7,81 & 4,66 & $\mathbf{2 4}$ & 12,90 & 5,40 & 3,28 & $\mathbf{3 4}$ & 15,85 & 6,54 & 3,75 \\
\hline $\mathbf{0 5}$ & $\mathbf{2 2}, 24$ & 8,70 & 4,75 & $\mathbf{1 5}$ & 14,60 & 5,66 & 3,13 & $\mathbf{2 5}$ & 25,81 & 10,14 & 5,81 & $\mathbf{3 5}$ & 19,58 & 7,35 & 4,08 \\
\hline $\mathbf{0 6}$ & 31,23 & 11,61 & 6,23 & $\mathbf{1 6}$ & 21,73 & 8,52 & 4,65 & $\mathbf{2 6}$ & 25,67 & 9,86 & 5,18 & $\mathbf{3 6}$ & 12,62 & 4,90 & 3,22 \\
\hline $\mathbf{0 7}$ & 22,56 & 9,24 & 6,09 & $\mathbf{1 7}$ & 19,15 & 9,55 & 5,85 & $\mathbf{2 7}$ & 23,20 & 8,65 & 5,47 & $\mathbf{3 7}$ & 24,49 & 8,76 & 4,55 \\
\hline $\mathbf{0 8}$ & $26, \mathbf{2 4}$ & 8,96 & 5,27 & $\mathbf{1 8}$ & 23,83 & 8,64 & 4,32 & $\mathbf{2 8}$ & 30,29 & 10,62 & 7,02 & $\mathbf{3 8}$ & 19,64 & 7,90 & 4,77 \\
\hline $\mathbf{0 9}$ & $\mathbf{2 0}, 50$ & 8,35 & 4,66 & $\mathbf{1 9}$ & 33,20 & 11,89 & 6,38 & $\mathbf{2 9}$ & 21,59 & 11,10 & 3,90 & & & & \\
\hline $\mathbf{1 0}$ & 13,42 & 7,01 & 3,62 & $\mathbf{2 0}$ & 23,84 & 9,31 & 4,93 & $\mathbf{3 0}$ & 23,26 & 9,72 & 6,36 & & & & \\
\hline
\end{tabular}

de cúpula (22,12 m), diámetro $(8,47 \mathrm{~m})$ y flecha $(4,84 \mathrm{~m})$; todos los valores obtenidos se recogen en la Tabla 2. La cúpula de mayor tamaño, tanto en altura como en diámetro, es la iglesia de la Virgen de Belén en Crevillente, con altura total de 33,20 m y diámetro de 11,89 m; valor que sí se aproxima a los 40 pies valencianos $(12,13 \mathrm{~m})$.

El diámetro medio de las cúpulas estudiadas presenta un valor inferior a los citados 40 pies; un dato bastante inferior al obtenido por García Jara (10) en 13,77 m de diámetro medio.

\subsection{Materiales de las cúpulas de crucero}

El ladrillo empleado es macizo y de dimensiones variables. En las muestras se distinguen dos tamaños predominantes: uno más grande de $30 \times 15 \times 3,5 \mathrm{~cm}$ y $2,5 \mathrm{~kg}$ de peso, y otro más pequeño de $26 \times 13 \times 3 \mathrm{~cm}$ y 1,5 $\mathrm{kg}$ de peso.

La cobertura en estas cúpulas se realiza con teja curva tanto para canales como para cobijas, encontrando algún caso aislado resuelto con canal de teja plana y cobija de teja curva. En las muestras obtenidas se distinguen dos tamaños: uno mayor en las cubiertas del templo, con dimensión aproximada de $43 \mathrm{~cm}$ de largo y $19 \mathrm{~cm}$ de ancho mayor; y otro menor, de $30 \mathrm{~cm}$ de largo y $15 \mathrm{~cm}$ de ancho mayor, empleado en la cubierta de la mayoría de las cúpulas y linternas.

En la mayoría de los templos $(68,42 \%)$, las cúpulas presentan tejas con acabado vidriado de color azul que contrasta con las tejas de limatesas en color blanco (5,26 \%), siendo ésta una característica propia en las cúpulas de la zona. También existen templos cubiertos con tejas de otros colores, como el marrón sin vidriar (18,42 \%) o el verde vidriado (7,90 \%).

Además, dada la inclinación de la cubierta, cabe destacar la importancia de los sistemas de anclaje mecánico de la última capa de tejas al soporte; en el caso de la provincia de Alicante se ejecuta mediante clavos metálicos (los más generalizados), clavos cerámicos (de mayor tamaño, con $6 \mathrm{~cm}$ de longitud y o,8 cm de diámetro) o de madera de olivo (bastante resistente aunque con menor durabilidad).

\section{CONCLUSIONES}

El diseño y la construcción de las cúpulas en los templos religiosos alicantinos desde finales del siglo XVII hasta principios del XIX son el resultado de la evolución en el ensayo de materiales y los avances de la técnica durante años. Una evolución donde, finalmente, se eligió el ladrillo como material principal, encontrando en el yeso un aliado perfecto. Así, tras el estudio en profundidad de la construcción, geometría y materiales en las cúpulas de la provincia cabe concluir:

\subsection{Sistemas constructivos de la cúpula}

Respecto a su forma de trabajo, los espesores máximos de ladrillo en la zona superior son del orden de 1/40 del radio de curvatura; aun así, para dar incluso mayor estabilidad, el empuje radial de las cúpulas se contrarresta mediante el relleno de enjutas con mortero de cal y cascotes, siempre simétricamente.

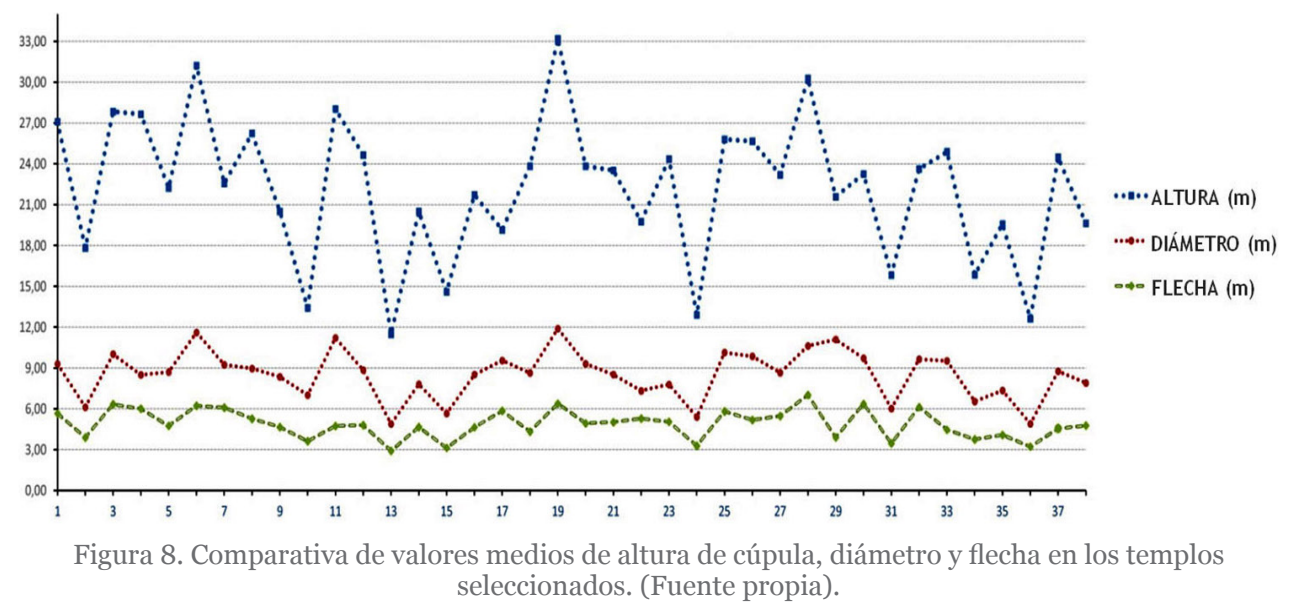



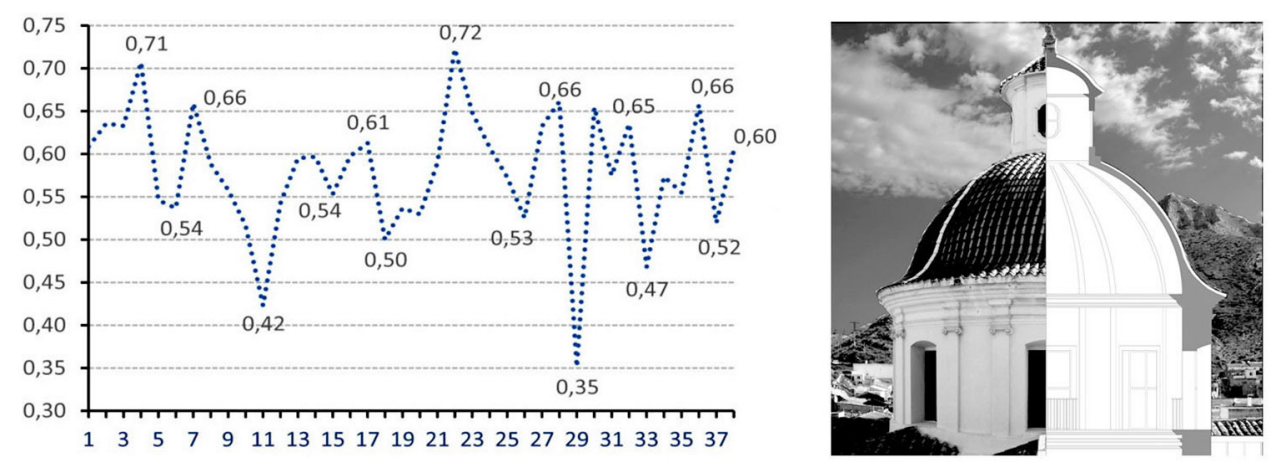

Figura 9. Izquierda. Relación entre flecha y diámetro en los templos seleccionados. (Fuente propia). Derecha: Fotomontaje de cúpula con sección. (Fuente propia).

De las 35 cúpulas simples incluidas en el estudio, el 88,57\% están compuestas por una sola hoja a rosca, al ser un sistema más económico (sin necesidad de cimbrado) y sencillo (mano de obra no especializada). Además, a esto hay que añadir que según el número de cáscaras, las cúpulas simples son las más abundantes en los templos de la provincia (92,11 \%) también por motivos económicos.

\subsection{Geometría de la cúpula}

En cuanto a su forma en planta, el 97,37 \% de los ejemplos presenta cúpulas de crucero de planta circular; respecto a su perfil, el 92,1\% de las cúpulas son peraltadas, curiosamente las más complejas de construir. El valor medio del peralte interior obtenido, es decir, la relación de flecha entre diámetro, es de 1/1,72 para los cruceros, valor inferior al mencionado por Fornés y Gurrea (2), donde recomienda una relación entre flecha y diámetro de $2 / 3$. No obstante, en algunos templos estudiados el peralte de la cúpula coincide con este último valor (Figura 9).

La necesidad de aumentar el espesor de la cúpula en su base, macizando su arranque o colocando lengüetas de ladrillo perpendiculares a la cúpula, junto con la necesidad de evacuación a los faldones de la cubierta inferior, dio como resultado un perfil característico en las cúpulas de la arquitectura religiosa alicantina: el perfil contracurvo.

$\mathrm{Al}$ interior, la cúpula queda revestida con yeso y está adornada mayoritariamente, en un 44,74\% de los casos, con 8 nervios decorativos, cuyo espesor varía de un templo a otro.

Respecto a las dimensiones generales de la cúpula, la altura total interior media en cruceros es $22,12 \mathrm{~m}$, el diámetro medio es $8,47 \mathrm{~m}$ y la flecha media es 4,84 $\mathrm{m}$. En algunos templos sorprenden las dimensiones construidas con ladrillo y yeso mediante cáscaras de poco espesor, siendo el valor máximo una altura de 33,20 $\mathrm{m}$ y un diámetro de 11,89 m.

\subsection{Materiales de la cúpula}

El ladrillo empleado es macizo en todos los casos, distinguiendo dos tamaños predominantes. El uso de ambos formatos en cada cúpula era muy útil en la ejecución de elementos abovedados, donde la forma curva hacía imprescindible piezas más pequeñas que suplieran el excesivo empleo de yeso. Dada la variedad de muestras obtenidas, se deduce que fueron fabricadas en distintas zonas geográficas cerca de cada templo y se ha constatado, en la composición de las fábricas, la recuperación de materiales de otras construcciones para su empleo en nuevos templos.

La cobertura de las cúpulas estudiadas se realiza con teja curva tanto para canales como para cobijas mediante dos tamaños principales. El 68,42 \% de tejas canales y cobijas presentan un acabado vidriado de color azul, con las limatesas resueltas con tejas curvas de color blanco, un rasgo característico e identificativo de las cúpulas de la provincia. Además, dada la inclinación de la cubierta, las tejas se colocan con fijación mecánica, empleando generalmente clavos metálicos.

Como conclusión final, cabe matizar que las cúpulas de ladrillo en los templos de la provincia de Alicante se generalizaron desde finales del siglo XVII hasta principios del XIX por su economía de puesta en obra, su facilidad de adaptación a cualquier forma y su menor peso en relación a su resistencia. Este material permitió reducir considerablemente espesores de muros y estribos, derivando en el desuso de cerchas para su puesta en obra (siendo esta nueva construcción más económica y de mayor rapidez de ejecución) y generando ciertas implicaciones tanto respecto a la forma de trabajo como respecto a las tipologías constructivas resultantes. Datos hasta ahora inexistentes e imprescindibles para establecer criterios de uso y conservación, facilitando futuras intervenciones de rehabilitación en estas construcciones religiosas.

\section{REFERENCIAS}

(1) San Nicolás, F.L. (1639). Arte y uso de arquitectura (Primera parte). Madrid.

(2) Fornés y Gurrea, M. (1841). Observaciones sobre la práctica del arte de edificar. Valencia: Imprenta de Cabrerizo.

(3) Le Duc, V. (1854-68). Dictionnaire raisonée de lárchitecture française du XIIe au XVIe siècle. Paris : Bance-Morel, traducción de la voz "Construction"

(4) Le Duc, V. (1996). La construcción medieval. Madrid. Instituto Juan de Herrera.

(5) Choisy, A. (1883). L'art de bâtir chez les byzantins. Paris: Librairie de la Société Anonyme de Publications Periodiques.

(6) Choisy, A. (1873). L'art de bâtir chez les romains. Paris: Librairie générale de l'architecture des travaux publics Ducher et Cie.

(7) Moya Blanco, L. (1947). Bóvedas tabicadas. Madrid: Colegio Oficial de Arquitectos de Madrid. 
(8) Truñó, A. (2004). Construcción de bóvedas tabicadas. Madrid: Instituto Juan de Herrera.

(9) Soler Verdú, R. (1995). La cúpula en la arquitectura moderna valenciana. Siglos XVI a XVIII. Metodologías de estudios previos, para las arquitecturas de sistemas abovedados (Tesis doctoral). Valencia: Universidad Politécnica de Valencia.

(10) García Jara, F. (2008). Las cúpulas de la arquitectura religiosa de la provincia de Alicante: del renacimiento al siglo $X I X$ (Tesis doctoral). Valencia: Universidad Politécnica de Valencia.

(11) Torija, J. (1661). Breve tratado de todo género de bóvedas. Madrid: Pablo del Val.

(12) Tosca, T.V. (1727). Tratado de la Montea y Cortes de Cantería. Segunda Impresión. Corregida, y enmendada de muchos yerros de Impresión, y laminas, como lo verá el curioso. Madrid: Imprenta de Antono Marín.

(13) Archivo Parroquial de San Juan Bautista de Cox. (1990). Documentación de la Restauración de la iglesia.

(14) Brizguz y Bru, A.G. (1738). Escuela de Arquitectura Civil, en que se contienen los ordenes de Arquitectura, la distribución de los Planos de Templos y Casas, y el conocimiento de los materiales. Valencia: Oficina de Joseph de Orga.

(15) Archivo Parroquial de los Santos Juanes de Catral. (1744). Capitulos y Condiciones del arrendamiento de la obra de la torre. Catral: Documentos de la Iglesia de Catral, G29.

(16) Fontana, C. (1694). Il Tempio Vaticano e sua origine. Roma: Stamparia di Gio, Francesco Buagni.

(17) Huerta, S. (2004). Arcos, bóvedas y cúpulas. Geometría y equilibrio en el cálculo tradicional de estructuras de fábrica. Madrid: Instituto Juan de Herrera. 